\title{
Estudo alométrico dos cortes de cordeiros Santa Inês puros e cruzas ${ }^{1}$ Iraides Ferreira Furusho-Garcia², Juan Ramón Olalquiaga Perez ${ }^{3}$, Sarita Bonagurio4, Cristiane Leal dos Santos 5
}

\author{
${ }^{1}$ Pesquisa financiada pela FAPEMIG. \\ 2 Universidade Federal dos Vales do Jequitinhonha e Mucuri. \\ 3 Universidade Federal de Lavras. \\ ${ }^{4}$ APTA - SP. \\ ${ }^{5}$ Universidade Estadual do Sudoeste da Bahia - Itapetinga - BA.
}

RESUMO - Cento e três cordeiros, machos e fêmeas, Santa Inês puros (SS) e cruzas com Texel (TS), Ile de France (FS) e Bergamácia (BS) foram utilizados no estudo do crescimento alométrico dos cortes. Os animais foram confinados em gaiolas individuais e abatidos aos 15, 25, 35 e $45 \mathrm{~kg}$ de PV. Após abate e resfriamento da carcaça, foram feitos os cortes: pescoço, costela/fralda, costeleta, lombo, paleta e perna, sendo esses dois últimos sem os braços, para avaliação do crescimento alométrico individual em relação ao corpo vazio (PCVZ). O crescimento alométrico do pescoço foi isogônico em todos os grupos, à exceção do grupo Santa Inês x Bergamácia (crescimento heterogônico positivo), comprovando que esse corte cresce de forma semelhante ao PCVZ. Os machos TS e FS apresentaram ritmo de crescimento do pescoço menor que o dos machos BS. A costela/fralda, em todos os grupos, teve crescimento lento entre os pesos avaliados, observando-se que, nos grupos TS e FS o ritmo de crescimento foi maior que no grupo SS. Nos machos, o desenvolvimento da paleta foi semelhante ao PCVZ, enquanto, nas fêmeas, foi lento em relação ao PCVZ. O desenvolvimento da costeleta e do lombo foi lento em todos os grupos, à exceção das fêmeas FS, nas quais acompanhou o PCVZ; os coeficientes alométricos de costeleta nesses animais foram menores que nos demais outros grupos. O desenvolvimento da perna foi similar ao PCVZ nos machos SS, TS e FS e nas fêmeas FS, porém, foi menos intenso nos machos BS e nas fêmeas SS, TS e BS.

Palavras-chave: carcaça, crescimento, desenvolvimento, ovinos

\section{Alometric study of cuts and tissues of the carcass of purebred and crossbred Santa Inês lambs}

\begin{abstract}
This study was conducted using 103 male and female lambs from the following genetic groups: purebred Santa Inês (SS) and crossbreds of Santa Inês x Texel (TS), Santa Inês x Ile de France (FS), and Santa Inês x Bergamacia (BS) Animals were slaughtered at one of the following body weights: 15, 25, 35 or $45 \mathrm{~kg}$. After slaughter and cooling of the carcasses, the following cuts were made: neck, rib/flank, short ribs, loin, blade, and hindlimb. The alometric growth of each cut in relation to the empty body weight (EBW) was measured. The alometric growth of the neck was isogonic ( $b=1)$ for most genetic groups and accompanied EBW growth. However, for BS lambs the growth was heterogonic $(b>1)$ or slow growth. TS and FS male lambs showed slower neck growth rate compared to the BS lambs. The rib/flank had slow growth for all groups but TS and FS lambs showed greater growth rate than the SS animals. For males, the blade development followed the EBW while females had a slower growth rate. The development of short ribs and loin was slow for all groups, except on FS female lambs, in which both short ribs and loin growth followed that of EBW; the alometric coefficients obtained for short ribs were lower on FS female lambs compared to those of the remaining genetic groups. The development of hindlimb accompanied the EBW for SS, TS and FS males and also for FS female lambs.
\end{abstract}

Key Words: growth, carcass, development, sheep

\section{Introdução}

O crescimento da ovinocultura de corte no país é eminente. Entretanto, os sistemas de produção e comercialização são desorganizados, constatando-se falta de uniformidade e de qualidade dos produtos para venda.
Com a franca expansão do mercado da carne ovina no Brasil, são necessárias pesquisas para determinação de pesos de abate e dos grupos genéticos mais adequados às condições nacionais, visando máxima eficiência produtiva e econômica para atender às exigências dos consumidores. 
A raça Santa Inês destaca-se no Brasil por seu elevado potencial para produção de carne magra e por sua utilização em cruzamentos com raças especializadas, possibilitando aumentar ainda mais a eficiência de produção. Entretanto, são poucos os estudos envolvendo a avaliação do desenvolvimento de animais resultantes desses cruzamentos, mais especificamente no aspecto qualitativo, cada vez mais importante para o consumidor.

A curva de crescimento dos animais geralmente é influenciada por fatores como raça, sexo, manejo alimentar, idade, maturidade, porte ou peso estabelecido (Sainz, 1996). O conhecimento do ritmo de crescimento de cada constituinte corporal, do ponto de vista econômico, pode possibilitar a determinação, com maior precisão, do peso ótimo de abate para cada grupo genético, viabilizando a máxima valorização do produto (Rocher et al., 1988; Silva et al., 2000b). Em virtude da alta velocidade de crescimento, o cordeiro é a categoria que fornece melhores rendimentos de carcaça e maior eficiência de produção (Pires et al., 2000). Entretanto, segundo Lohose et al. (1971) e Rocher \& Espejo (1973), citados por Silva et al. (2000a), os componentes ou cortes da carcaça, como paleta, perna e costelas, podem apresentar desenvolvimento tardio ou precoce ou acompanhar o desenvolvimento corporal.

Assim, é importante a avaliação do crescimento de animais adaptados às condições de produção no Brasil, principalmente para determinação da fase do desenvolvimento em que se obtém um produto de qualidade e que promova melhores retornos econômicos.

Como existem fases do crescimento em que determinadas partes da carcaça se desenvolvem mais intensamente e considerando a existência de cortes de "primeira" e de "segunda", é interessante identificar quando podem ser obtidas melhores proporções, sobretudo dos cortes nobres. Segundo Hammond (1966), a velocidade de crescimento de cada região corporal avança até alcançar o máximo e começa a decrescer à medida que o animal se aproxima do tamanho adulto.

O estudo alométrico do crescimento permite estimar o padrão de desenvolvimento dos cortes de importância econômica nos animais. De acordo com Ávila \& Osório (1996), o estudo da alometria está baseado, principalmente, no fato de o desenvolvimento corporal ser uma função do peso e não do tempo necessário para alcançá-lo. Por isso, o abate de animais com determinada amplitude de variação de peso vivo, realizado seqüencialmente, tem sido um dos métodos mais utilizados para descrever o crescimento relativo dos componentes da carcaça (Berg \& Butterfield, 1966).
Segundo Santos (1999), a alometria, ao explicar parte das diferenças quantitativas entre os animais, pode ser um parâmetro eficaz no estudo da carcaça e de seus componentes. O estudo alométrico proporciona descrição quantitativa da relação entre uma parte e o todo e, apesar de não registrar detalhes, é importante por agregar todas as informações em um só valor (Berg \& Butterfield, 1966).

Neste estudo, avaliou-se o crescimento alométrico dos cortes de cordeiros, machos e fêmeas, Santa Inês puros e cruzas com Texel, Ile de France e Bergamácia, terminados em confinamento.

\section{Material e Métodos}

O experimento foi realizado no Setor de Ovinocultura do Departamento de Zootecnia da Universidade Federal de Lavras. Foram utilizados 103 cordeiros, machos e fêmeas, resultantes de acasalamentos entre ovelhas Santa Inês e reprodutores Santa Inês (SS), Texel (TS), Ile de France (FS) e Bergamácia (BS). Todos os cordeiros (27 SS, 27 TS, 18 FS e 32 BS) nasceram entre junho e julho de 1999 e foram desmamados aos 10-12 kg de PV, sendo então confinados em grupo e alimentados com a dieta experimental até atingirem $14 \mathrm{~kg}$ de PV. Em seguida, foram confinados em gaiolas individuais $\left(1,3 \mathrm{~m}^{2}\right)$ providas de cochos e bebedouros separados.

A dieta fornecida (à vontade) durante todo o período experimental foi balanceada de acordo com as exigências do ARC (1988) para proporcionar ganho de $300 \mathrm{~g} / \mathrm{dia}$, sendo composta de $80 \%$ de concentrado e $20 \%$ de feno de coastcross (Cynodon dactylon) moído (Tabela 1).

Após o desmame, os animais (machos e fêmeas) de cada grupo genético foram escolhidos aleatoriamente para cada peso de abate pré-estabelecido: 15, 25, 35 e $45 \mathrm{~kg}$ de PV. O abate foi realizado após jejum de sólidos (16 horas), nos animais suspensos pelos membros posteriores, por meio de atordoamento e secção da artéria carótida e das veias jugulares. O sangue e a pele foram coletados e pesados e as vísceras retiradas para obtenção do peso corporal vazio (PCVZ) e do peso de carcaça quente (PCQ).

As carcaças foram mantidas durante 24 horas em câmara fria $\left(2 \mathrm{a} 4^{\circ} \mathrm{C}\right)$, sendo novamente pesadas para determinação do peso de carcaça fria (PCF). Depois de retirados o pescoço, a cauda, os rins e as gorduras renais, pélvica e inguinal, a carcaça foi dividida longitudinalmente em duas metades.

A metade esquerda foi subdividida em perna, lombo, paleta, costeleta, costela/fralda, braço anterior e braço posterior (Santos, 1999), como ilustrado na Figura 1. Os cortes foram acondicionados em sacos plásticos e mantidos em freezer até o momento da dissecação, realizada após 
Tabela 1 - Composição da dieta experimental (\% na matéria natural)

Table 1 - Ingredient composition of the experimental diet (\% as-fed basis)

\begin{tabular}{lc}
\hline $\begin{array}{l}\text { Ingrediente (\%) } \\
\text { Ingredient }\end{array}$ & $\begin{array}{c}\text { Base da matéria natural } \\
\text { As-fed basis (\%) }\end{array}$ \\
\hline Feno de coast cross (Coast cross hay) & 20,00 \\
Milho moído (Ground corn) & 66,45 \\
Farelo de soja (Soybean meal) & 12,40 \\
Calcário (Limestone) & 0,85 \\
Sal (Salt) & 0,25 \\
Suplemento mineral ${ }^{\text {a }}$ (Mineral supplement) & 0,01 \\
Suplemento vitamínico $^{\text {b }}$ (Vitamin supplement) & 0,04
\end{tabular}

a (nutriente/kg de suplemento) (nutrient $/ \mathrm{kg}$ of supplement): $\mathrm{Se}=150 \mathrm{mg}, \mathrm{I}=1.000$ $\mathrm{mg}, \mathrm{Co}=600 \mathrm{mg}, \mathrm{Fe}=35.000 \mathrm{mg}, \mathrm{Cu}=20.000 \mathrm{mg}, \mathrm{Mn}=49.000 \mathrm{mg}$, $\mathrm{Zn}=75.000 \mathrm{mg}$.

b (nutriente/kg de suplemento) (nutrient $/ \mathrm{kg}$ of supplement): Vit. A $=2.500 .000 \mathrm{UI}$, Vit. D3 $500.000 \mathrm{UI}$, Vit. E $3.000 \mathrm{mg}$, Tiamina (Tiamin) $=750 \mathrm{mg}$, Riboflavina $($ Riboflavin $)=1.000 \mathrm{mcg}$, Vit. B12 $=2.800$, Niacina $($ Niacin $)=500 \mathrm{mg}$.

o descongelamento dos cortes em temperatura ambiente. De cada corte foram separados os tecidos muscular, ósseo e adiposo (gordura subcutânea + intermuscular) e outros (veias, artérias, tendões, tecido conjuntivo e sangue coagulado).

Realizou-se o estudo do crescimento dos cortes em relação ao do corpo vazio. Para estudo do crescimento alométrico, foi utilizado o modelo de equação exponencial, $\mathrm{Y}_{\mathrm{i}}=\mathrm{a} \mathrm{X}_{\mathrm{i}}^{\mathrm{b}} \varepsilon_{\mathrm{i}}$, transformado de forma logarítmica em um modelo linear (Huxley, 1932):

$$
\ln \mathrm{Y}_{\mathrm{i}}=\ln \mathrm{a}+\mathrm{b} \ln \mathrm{X}+\ln \varepsilon_{\mathrm{i}}
$$

em que: $\mathrm{Y}_{\mathrm{i}}=$ peso do corte ou do tecido; $\mathrm{X}=$ peso do corpo vazio, do corte ou da carcaça fria; $a=$ intercepção do logaritmo da regressão linear sobre "Y" e "b"; b = coeficiente de crescimento relativo ou de alometria; $\varepsilon_{\mathrm{i}}=$ erro multiplicativo; $\mathrm{Ln}=$ logaritmo neperiano.

Os dados foram analisados pelo procedimento REG do programa Statistical Analysis System (SAS, 1995), aplicando-se o teste $T$ para verificação da hipótese de $\mathrm{H}_{0}$ : $\beta=1$. $\mathrm{O}$ crescimento foi considerado isogônico quando $\mathrm{b}=1$, indicando taxas de desenvolvimento de " $X$ " e " $Y$ " semelhantes no intervalo de crescimento avaliado. No caso de $b \neq 1,0$ crescimento foi considerado heterogônico, sendo positivo quando $b>1$ e negativo quando $b<1$. Os coeficientes de alometria foram testados por meio do teste $F$ para comparação dos grupos genéticos, utilizando-se variável binária (Graybill, 1976; Regazzi \& Leite, 1992; Regazzi, 1993) para identificação dos coeficientes de regressão de cada grupo genético.

\section{Resultados e Discussão}

Os coeficientes de alometria, $\mathrm{R}^{2}$ e os valores de probabilidade do teste $\mathrm{F}$ para a comparação destes coeficientes do pescoço nos animais machos e fêmeas entre os grupos

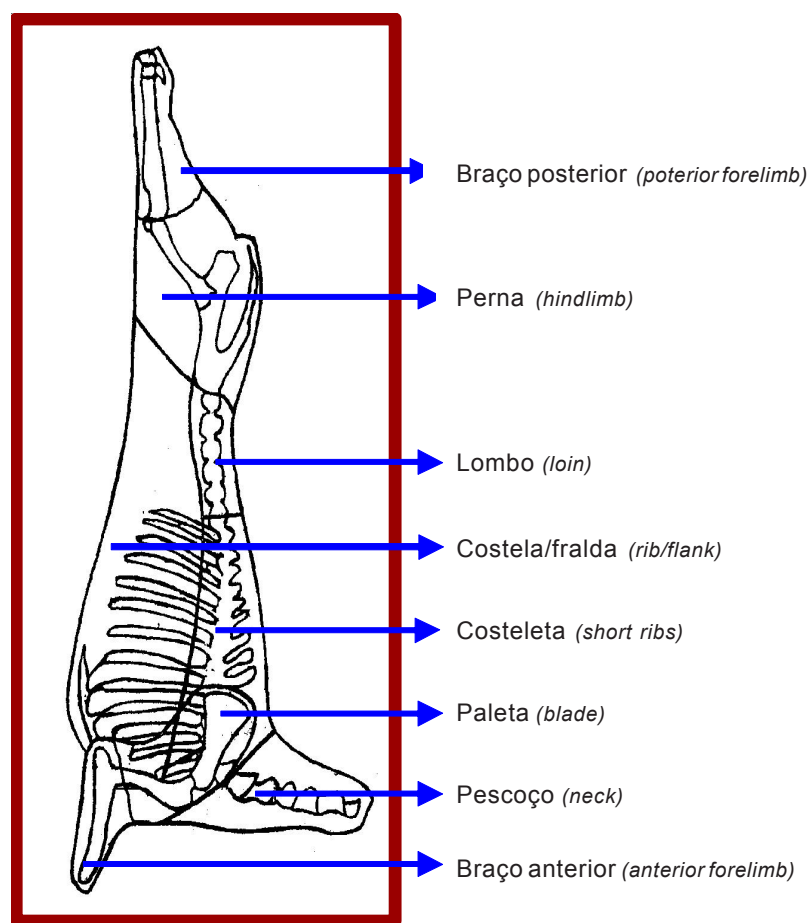

Figura 1 - Cortes efetuados na meia-carcaça esquerda. Figure 1 - Cuts done in the $1 / 2$ left carcass.

genéticos são apresentados na Tabela 2. Os animais do grupo BS apresentaram coeficientes alométricos diferentes de $1(\mathrm{P}<0,01)$, que caracterizam crescimento heterogônico positivo, com menor intensidade de desenvolvimento do pescoço em relação ao PCVZ nesse grupo genético, enquanto, nos demais, o desenvolvimento foi isogônico ( $\mathrm{P}>0,05)$ e acompanhou o corpo vazio.

Os resultados do teste $\mathrm{F}$ demonstraram que os machos BS apresentaram ritmo de crescimento do pescoço em relação ao corpo vazio maior que o dos cordeiros dos grupos TS e FS $(\mathrm{P}<0,05)$. Desta forma, os animais cruzas $\mathrm{BS}$, apesar do lento desenvolvimento lento do pescoço, apresentaram maior ritmo de crescimento desse corte. Este resultado é coerente com as características de uma raça de grande porte, como a Bergamácia. Nas fêmeas, o ritmo de crescimento não diferiu entre os grupos $(\mathrm{P}>0,05)$.

De acordo com os coeficientes alométricos de machos e fêmeas de todos os grupos genéticos, o crescimento do corte costela/fralda foi heterogônico positivo $(\mathrm{P}<0,05)$, indicando menor intensidade de desenvolvimento desse corte (Tabela 3). Resultados semelhantes foram relatados por Silva et al. (2000b) para regiões corporais semelhantes.

Pesquisadores (Lohose et al., 1971; Santos, 1999; Silva \& Portugal, 2000) afirmam que a costela/fralda, por fazer parte de uma região corporal que se desenvolve mais tardiamente, cresce à medida que aumenta o peso do animal. 
Tabela 2 - Coeficientes de alometria comparados pelo teste $t$, coeficientes de determinação $\left(R^{2}\right)$ e valores de probabilidade do teste $F$ para pescoço de machos e fêmeas Santa Inês puros (SS) e cruzas com Texel (TS), Ile de France (FS) e Bergamácia (BS)

Table 2 - Comparisons of alometric coefficients ( $t$ test), coefficients of determination $\left(R^{2}\right)$, and differences ( $F$ test) among genetic groups for the neck of male and female purebred Santa Inês (SS) and crossbreds of Texel x Santa Inês (TS), Ile de France x Santa Inês (FS) and Bergamácia x Santa Inês (BS) lambs

\begin{tabular}{|c|c|c|c|c|c|c|c|}
\hline \multirow{2}{*}{$\begin{array}{l}\text { Grupo genético } \\
\text { Genetic group }\end{array}$} & \multirow{2}{*}{$\begin{array}{l}\text { Coeficiente de alometria } \\
\text { Alometric coefficient }\end{array}$} & \multirow[t]{2}{*}{$\mathrm{R}^{2}$} & \multirow{2}{*}{$\begin{array}{l}\text { Teste } t \\
\text { Ho: } \beta=1\end{array}$} & \multicolumn{4}{|c|}{ Prob: Teste F } \\
\hline & & & & SS & TS & FS & BS \\
\hline \multicolumn{8}{|l|}{ Machos (Males) } \\
\hline SS & 1,1604 & 0,9534 & ns & - & 0,1424 & 0,3861 & 0,1965 \\
\hline TS & 0,9973 & 0,9294 & ns & - & - & 0,6728 & 0,0068 \\
\hline FS & 1,0497 & 0,9453 & $\mathrm{~ns}$ & - & - & - & 0,0473 \\
\hline BS & 1,3080 & 0,9667 & $* *$ & - & - & - & - \\
\hline \multicolumn{8}{|l|}{ Fêmeas (Females) } \\
\hline SS & 1,0276 & 0,9624 & ns & - & 0,8919 & 0,5499 & 0,0847 \\
\hline TS & 1,0087 & 0,7807 & ns & - & - & 0,4722 & 0,0607 \\
\hline FS & 1,1228 & 0,9766 & ns & - & - & - & 0,3870 \\
\hline BS & 1,2522 & 0,9577 & $* *$ & - & - & - & - \\
\hline
\end{tabular}

${ }^{* *} \mathrm{P}<0,01 ;{ }^{*} \mathrm{P}<0,05$

Tabela 3 - Coeficientes de alometria comparados à unidade pelo teste t, coeficientes de determinação $\left(R^{2}\right)$ e valores de probabilidade do teste F para costela/fralda de cordeiros, machos e fêmeas, Santa Inês puros (SS) e cruzas com Texel (TS), Ile de France (FS) e Bergamácia (BS)

Table 3 - Comparisons of alometric coefficients ( $t$ test), coefficients of determination $\left(R^{2}\right)$, and differences ( $F$ test) among genetic groups for the rib/flank of male and female purebred Santa Inês (SS) and crossbreds of Texel x Santa Inês (TS), Ile de France x Santa Inês (FS) and Bergamácia x Santa Inês (BS) lambs

\begin{tabular}{|c|c|c|c|c|c|c|c|}
\hline \multirow{2}{*}{$\begin{array}{l}\text { Grupo genético } \\
\text { Genetic group }\end{array}$} & \multirow{2}{*}{$\begin{array}{c}\text { Coeficiente de alometria } \\
\text { Alometric coefficient }\end{array}$} & \multirow[t]{2}{*}{$\mathrm{R}^{2}$} & \multirow{2}{*}{$\begin{array}{l}\text { Teste } \mathrm{t} \\
\text { Ho: } \beta=1\end{array}$} & \multicolumn{4}{|c|}{ Prob: Teste F } \\
\hline & & & & SS & TS & FS & BS \\
\hline \multicolumn{8}{|l|}{ Machos (Males) } \\
\hline SS & 1,3207 & 0,9842 & $* *$ & - & 0,7309 & 0,4144 & 0,2117 \\
\hline TS & 1,2967 & 0,9832 & $* *$ & - & - & 0,5927 & 0,3413 \\
\hline FS & 1,2538 & 0,9705 & $*$ & - & - & - & 0,7629 \\
\hline BS & 1,2302 & 0,9935 & $* *$ & - & - & - & - \\
\hline \multicolumn{8}{|l|}{ Fêmeas (Females) } \\
\hline SS & 1,2023 & 0,9567 & $*$ & - & 0,0096 & 0,0385 & 0,1934 \\
\hline TS & 1,4161 & 0,9825 & $* *$ & - & - & 0,8181 & 0,1126 \\
\hline FS & 1,3952 & 0,9899 & $* *$ & - & - & - & 0,2616 \\
\hline BS & 1,2988 & 0,9862 & $* *$ & - & - & - & - \\
\hline
\end{tabular}

${ }^{* *} \mathrm{P}<0,01 ;{ }^{*} \mathrm{P}<0,05$.

Os valores de probabilidade do teste F para a comparação dos coeficientes de alometria da costela/fralda entre os grupos genéticos (Tabela 3) indicaram não haver diferenças entre estes coeficientes para o ritmo de crescimento da costela/fralda dos machos ( $\mathrm{P}>0,05)$. As fêmeas SS apresentaram ritmo de crescimento menor para este corte, em comparação às TS e FS, evidenciando o efeito do cruzamento neste corte $(\mathrm{P}<0,05)$.

Ao contrário do pescoço e da fralda/costela, o crescimento da paleta foi isogônico $(\mathrm{P}>0,05)$ e heterogônico $(\mathrm{P}<0,05)$ para machos e fêmeas (Tabela 4), respectivamente. Os machos de todos os grupos genéticos estudados apre- sentaram coeficientes de alometria iguais a 1 , ou seja, crescimento isogônico em relação ao PCVZ, o que está de acordo com o resultado encontrado por Santos (1999), que estudou o crescimento de cordeiros machos Santa Inês e Bergamácia.

As fêmeas de todos os grupos genéticos apresentaram coeficientes de alometria inferiores a $1(\mathrm{P}<0,05)$, refletindo um crescimento heterogônico positivo, ou seja, desenvolvimento em menor intensidade em relação ao PCVZ. Esse resultado está associado ao fato de as fêmeas depositarem mais gordura nessa região, como constatado naquelas abatidas aos $45 \mathrm{~kg}$ (Furusho-Garcia, 2001). Por ser um tecido de crescimento mais tardio, a gordura influenciou o desen- 
volvimento da paleta, resultando em desenvolvimento mais intenso desse corte somente no período final de crescimento. Os resultados encontrados, tanto para machos como para fêmeas, divergem dos citados por Lohose et al. (1971) e Silva et al. (2000b), que encontraram para paleta um desenvolvimento mais intenso.

$\mathrm{Na}$ Tabela 4 são apresentados os valores de probabilidade do teste $\mathrm{F}$ para machos e fêmeas, entre os grupos genéticos, para a comparação dos coeficientes de alometria para paleta. Os dados comprovam que, tanto para machos como para fêmeas, o ritmo de crescimento de paleta não diferiu entre os grupos genéticos $(\mathrm{P}>0,05)$.

Os dados referentes ao crescimento alométrico da costeleta são apresentados na Tabela 5, juntamente com os valores do teste $\mathrm{F}$ e a comparação dos coeficientes alométricos dos diferentes grupos genéticos.

De acordo com os coeficientes de alometria das equações, tanto machos como fêmeas tiveram crescimento heterogônico positivo $(\mathrm{P}>0,05)$, que indica desenvolvimento menos intenso para esse corte, à exceção das fêmeas do grupo FS, que mostraram crescimento isogônico $(\mathrm{P}>0,05)$, ou seja, a costeleta teve desenvolvimento semelhante ao PCVZ. Os dados deste estudo assemelham-se àqueles descritos por Silva et al. (2000b), que citaram que a costela possui desenvolvimento mais lento. Ressalta-se que, neste trabalho, a costeleta não foi analisada junto com as costelas.

Os valores de probabilidade do teste F obtidos na comparação dos coeficientes de alometria da costela/fralda dos

Tabela 4 - Coeficientes de alometria comparados pelo teste $t$, coeficientes de determinação $\left(R^{2}\right)$ e valores de probabilidade do teste $F$, para paleta de cordeiros, machos e fêmeas, Santa Inês puros (SS) e cruzas com Texel (TS), lle de France (FS) e Bergamácia (BS)

Table 4 - Comparisons of alometric coefficients ( $t$ test), coefficients of determination $\left(R^{2}\right)$, and differences ( $F$ test) among genetic groups for the blade of male and female purebred Santa Inês (SS) and crossbreds of Texel x Santa Inês (TS), Ile de France x Santa Inês (FS) and Bergamácia x Santa Inês (BS) lambs

\begin{tabular}{|c|c|c|c|c|c|c|c|}
\hline $\begin{array}{l}\text { Grupo genético } \\
\text { Genetic group }\end{array}$ & $\begin{array}{c}\text { Coeficiente de alometria } \\
\text { Alometric coefficient }\end{array}$ & $\mathrm{R}^{2}$ & $\begin{array}{l}\text { Teste } \mathrm{t} \\
\text { Ho: } \beta=1\end{array}$ & \multicolumn{4}{|c|}{ Prob: Teste F } \\
\hline \multicolumn{8}{|l|}{ Machos (Males) } \\
\hline SS & 1,0741 & 0,9744 & ns & - & 0,7577 & 0,7107 & 0,4378 \\
\hline TS & 1,0533 & 0,9769 & ns & - & - & 0,5144 & 0,6214 \\
\hline FS & 1,1030 & 0,9764 & ns & - & - & - & 0,2902 \\
\hline SS & 1,2043 & 0,977 & $* *$ & - & 0,3527 & 0,4246 & 0,0919 \\
\hline $\mathrm{TS}$ & 1,1458 & 0,9785 & $*$ & - & - & 0,9883 & 0,4821 \\
\hline FS & 1,1468 & 0,9865 & $*$ & - & - & - & 0,5374 \\
\hline BS & 1,1052 & 0,9902 & $* *$ & - & - & - & - \\
\hline
\end{tabular}

${ }^{* *} \mathrm{P}<0,01 ;{ }^{*} \mathrm{P}<0,05$.

Tabela 5 - Coeficientes de alometria comparado pelo teste t, coeficientes de determinação $\left(R^{2}\right)$ e valores de probabilidade do teste $F$ para costeleta de machos e fêmeas Santa Inês puros (SS) e cruzas com Texel (TS), lle de France (FS) e Bergamácia (BS)

Table 5 - Comparisons of alometric coefficients ( $t$ test), coefficients of determination $\left(R^{2}\right)$, and differences ( $F$ test) among genetic groups for the short ribs of male and female purebred Santa Inês (SS) and crossbreds of Texel x Santa Inês (TS), Ile de France x Santa Inês (FS) and Bergamácia x Santa Inês (BS) lambs

\begin{tabular}{|c|c|c|c|c|c|c|c|}
\hline $\begin{array}{l}\text { Grupo genético } \\
\text { Genetic group }\end{array}$ & $\begin{array}{l}\text { Coeficiente de alometria } \\
\text { Alometric coefficient }\end{array}$ & $\mathrm{R}^{2}$ & $\begin{array}{c}\text { Teste } \mathrm{t} \\
\text { Ho: } \beta=1\end{array}$ & \multicolumn{4}{|c|}{ Prob: Teste F } \\
\hline \multicolumn{8}{|l|}{ Machos (Males) } \\
\hline SS & 1,2480 & 0,9726 & $* *$ & - & 0,5135 & 0,9618 & 0,6076 \\
\hline TS & 1,1897 & 0,9752 & $* *$ & - & - & 0,5305 & 0,9000 \\
\hline FS & 1,2530 & 0,9812 & $* *$ & - & - & - & 0,6135 \\
\hline SS & 1,3087 & 0,9452 & $* *$ & - & 0,5558 & 0,0053 & 0,7097 \\
\hline TS & 1,2504 & 0,9908 & $* *$ & - & - & 0,0195 & 0,7884 \\
\hline FS & 0,9795 & 0,9668 & $\mathrm{~ns}$ & - & - & - & 0,0074 \\
\hline BS & 1,2747 & 0,9616 & $* *$ & - & - & - & - \\
\hline
\end{tabular}

** $P<0,01 ;{ }^{*} P<0,05$. 
grupos genéticos (Tabela 5) indicaram que nos machos os ritmos de crescimento, com menor intensidade, da costeleta foram semelhantes entre si e que as fêmeas do grupo FS que apresentaram crescimento isogônico diferiram daquelas fêmeas dos demais grupos genéticos, indicando desenvolvimento relativamente lento dessa região corporal.

Os coeficientes alométricos para o desenvolvimento do lombo e os valores de probabilidade do teste F para a comparação destes coeficientes, entre os grupos genéticos, para machos e fêmeas, são apresentados na Tabela 6 .

Machos e fêmeas dos grupos genéticos SS, TS e BS apresentaram coeficientes de alometria diferentes de 1 $(\mathrm{P}<0,05)$, que indica crescimento heterogônico positivo e desenvolvimento menos intenso para o lombo, de modo semelhante ao citado por Berg \& Butterfield (1976). Nos cordeiros do grupo FS, machos e fêmeas, o crescimento foi isogônico ( $\mathrm{P}>0,05)$, com crescimento semelhante ao PCVZ, como observado também por Santos (1999), em cordeiros Santa Inês e Bergamácia.

Os valores de probabilidade do teste $\mathrm{F}$ permitem inferir que machos e fêmeas dos diferentes os grupos genéticos $(\mathrm{P}>0,05)$ apresentaram ritmos de crescimento tardio semelhantes entre si.

Na Tabela 7 são apresentados os coeficientes alométricos e os valores de probabilidade do teste $\mathrm{F}$, respectivamente, de machos e fêmeas, para a comparação dos coeficientes de alometria da perna entre os grupos genéticos.

Os machos do grupo BS apresentaram crescimento heterogônico positivo $(\mathrm{P}<0,05)$, caracterizando desenvolvimento lento para esse corte, enquanto os demais grupos apresentaram crescimento isogônico $(\mathrm{P}>0,05)$, com desen- volvimento semelhante ao PCVZ. As fêmeas dos grupos SS, TS eBS apresentaram crescimento heterogônico $(P<0,05)$ e as FS crescimento isogônico $(b=1)$. O desenvolvimento tardio da perna apresentado por alguns animais neste trabalho, não está de acordo com o observado por Lohose et al. (1971), Colomer \& Espejo (1973), citados por Osório et al. (1995) e Silva et al. (2000b), que afirmaram que a região do quarto posterior possui desenvolvimento menos intenso. Santos (1999) encontrou, para a perna de cordeiros Santa Inês e Bergamácia, crescimento isogônico.

$\mathrm{O}$ fato de os cordeiros BS e fêmeas SS e TS apresentarem crescimento com menor intensidade para a perna pode ser atribuído à tendência de os animais do grupo BS depositarem mais gordura que os outros genótipos, como verificado para as fêmeas SS e TS, que depositaram mais na perna, contribuindo para um desenvolvimento menos intenso desse corte.

Os valores de probabilidade do teste F, obtidos pela comparação da velocidade de crescimento da perna dos diferentes grupos genéticos, indicaram taxas de crescimento, tanto para machos como para fêmeas, similares entre os grupos $(\mathrm{P}>0,05)$, demonstrando que as diferenças genéticas entre os animais não interferem na taxa de crescimento dessa região da carcaça.

\section{Conclusões}

Cordeiros Santa Inês puros e cruzas com Texel, Ile de France e Bergamácia possuem ritmo de desenvolvimento do pescoço semelhante ao do corpo vazio. A costela/fralda, a costeleta e o lombo desenvolvem-se mais lentamente em relação ao corpo vazio.

Tabela 6 - Coeficientes de alometria comparados pelo teste t, coeficientes de determinação $\left(R^{2}\right)$ e valores de probabilidade do teste $F$ para o lombo de machos e fêmeas Santa Inês puros (SS) e cruzas com Texel (TS), Ile de France (FS) e Bergamácia (BS)

Table 6 - Comparisons of alometric coefficients ( $t$ test), coefficients of determination $\left(R^{2}\right)$, and differences (Ftest) among genetic groups for the loin of male and female purebred Santa Inês (SS) and crossbreds of Texel x Santa Inês (TS), Ile de France x Santa Inês (FS) and Bergamácia x Santa Inês (BS) lambs

\begin{tabular}{|c|c|c|c|c|c|c|c|}
\hline $\begin{array}{l}\text { Grupo genético } \\
\text { Genetic group }\end{array}$ & $\begin{array}{c}\text { Coeficiente de alometria } \\
\text { Alometric coefficient }\end{array}$ & $\mathrm{R}^{2}$ & $\begin{array}{l}\text { Teste } t \\
\text { Ho: } \beta=1\end{array}$ & \multicolumn{4}{|c|}{ Prob: Teste $\mathrm{F}$} \\
\hline SS & 1,1874 & 0,9589 & $*$ & - & 0,7953 & 0,7309 & 0,4820 \\
\hline TS & 1,2190 & 0,9601 & $*$ & - & - & 0,9019 & 0,6403 \\
\hline FS & 1,2359 & 0,8756 & ns & - & - & - & 0,7773 \\
\hline
\end{tabular}

Fêmeas (Females)

\begin{tabular}{lcccccc} 
SS & 1,4035 & 0,9885 & $* *$ & - & 0,6530 & 0,0870 \\
TS & 1,3621 & 0,9643 & $* *$ & - & - & 0,3084 \\
FS & 1,2205 & 0,958 & ns & - & - & - \\
BS & 1,3165 & 0,9751 & $* *$ & - & - & - \\
\hline
\end{tabular}

** $P<0,01 ;{ }^{*} P<0,05$. 
Tabela 7 - Coeficientes de alometria comparados pelo teste t, coeficientes de determinação $\left(R^{2}\right)$ e valores de probabilidade do teste $F$ para a perna de machos e fêmeas Santa Inês puros (SS) e cruzas com Texel (TS), lle de France (FS) e Bergamácia (BS)

Table 7 - Comparisons of alometric coefficients ( $t$ test), coefficients of determination $\left(R^{2}\right)$, and differences ( $F$ test) among genetic groups for the hindlimb of male and female purebred Santa Inês (SS) and crossbreds of Texel x Santa Inês (TS), Ile de France x Santa Inês (FS) and Bergamácia x Santa Inês (BS) lambs

\begin{tabular}{|c|c|c|c|c|c|c|c|}
\hline \multirow{2}{*}{$\begin{array}{l}\text { Grupo genético } \\
\text { Genetic group }\end{array}$} & \multirow{2}{*}{$\begin{array}{c}\text { Coeficiente de alometria } \\
\text { Alometric coefficient }\end{array}$} & \multirow[t]{2}{*}{$\mathrm{R}^{2}$} & \multirow{2}{*}{$\begin{array}{l}\text { Teste } \mathrm{t} \\
\text { Ho: } \beta=1\end{array}$} & \multicolumn{4}{|c|}{ Prob: Teste F } \\
\hline & & & & SS & TS & FS & BS \\
\hline \multicolumn{8}{|l|}{ Machos (Males) } \\
\hline SS & 1,0172 & 0,9847 & ns & - & 0,4446 & 0,6740 & 0,4045 \\
\hline TS & 0,9756 & 0,9749 & ns & - & - & 0,2704 & 0,1081 \\
\hline FS & 1,0437 & 0,9816 & $\mathrm{~ns}$ & - & - & - & 0,7465 \\
\hline BS & 1,0640 & 0,996 & $*$ & - & - & - & - \\
\hline \multicolumn{8}{|l|}{ Fêmeas (Females) } \\
\hline SS & 1,0871 & 0,9848 & $* *$ & - & 0,6357 & 0,6072 & 0,8099 \\
\hline TS & 1,1117 & 0,9922 & $* *$ & - & - & 0,3529 & 0,7835 \\
\hline FS & 1,0565 & 0,9958 & $\mathrm{~ns}$ & - & - & - & 0,4509 \\
\hline BS & 1,0986 & 0,982 & $* *$ & - & - & - & - \\
\hline
\end{tabular}

${ }^{* *} \mathrm{P}<0,01 ;{ }^{*} \mathrm{P}<0,05$

\section{Literatura Citada}

AGRICULTURAL RESEARCH COUNCIL - ARC. The nutrient requeriment of ruminant livestock. London: Lavenham Press Ltda., 1988. 351p.

ÁVILA, V.S.; OSÓRIO, J.C.S. Efeito do sistema de criação, época de nascimento e ano na velocidade de crescimento de cordeiros. Revista da Sociedade Brasileira de Zootecnia, v.25, n.5, p.1007-1016, 1996.

BERG, R.T.; BUTTERFIELD, R.M. Muscle: bone ratio and fat percentage as measures of beef carcase composition. Animal Production, v.8, n.1, p.1-11, 1966.

BERG, R.T.; BUTTERFIELD, R.M. New concepts of cattle growth. Sydney: Sydney University Press, 1976. 240p.

FURUSHO-GARCIA, I.F. Desempenho, caracterização da carcaça, alometria dos cortes e tecidos e eficência da energia, em cordeiros Santa Inês e cruzas com Texel, Ile de France e Bergamácia. Lavras: Universidade Federal de Lavras, 2001. 316p. Tese (Doutorado em Zootecnia) Universidade Federal de Lavras, 2001.

GRAYBILL, F.A. Theory and application of the linear model. Massachusetts: Duxbury Press, 1976. 704p.

HAMMOND, J. Reprodución, crecimiento y herancia. In: Principios de la explotación animal. Zaragoza: Acribia, 1966. p.142-157.

HUXLEY, J.S. Problems of relative growth. London: Methuen, 1932. 276p.

LOHOSE, C.L.; MOSS, F.P.; BUTTERFIELD, R.M. Growth patterns of muscle of Merino sheep from birth to 517 days. Animal Production, v.13, n.1, p.117-126, 1971.

OSÓRIO, J.C.S.; SIEWERDT, F.; OSÓRIO, M.T.M. et al. Desenvolvimento alométrico das regiões corporais em ovinos. Revista da Sociedade Brasileira de Zootecnia, v.24, n.2, p.326-333, 1995.

PIRES, C.C.; SILVA, L.F.; SCHLICK, F.E. et al. Cria e terminação de cordeiros confinados. Ciência Rural, v.30, n.5, p.875-880, 2000.

REGAZZI, A.J. Teste para verificar a identidade de modelos de regressão e a igualdade de alguns parâmetros num modelo polinomial ortogonal. Revista Ceres, v.40, n.228, p.176-195, 1993.

RegaZZI, A.J.; LEITE, H.G. Análise de regressão: Teoria e aplicações em manejo florestal. Viçosa, MG: Universidade Federal de Viçosa, 1992. 236p. (Texto acadêmico)
ROCHER, F.C.; DELAT, R.; SIERRA-ALFRANCA, I. Métodos normalizados para el estúdio de los caracteres cuantitativos e cualitativos de las canales caprinas $y$ ovinas. In:_ Método normalizado para el studio de los caracteres cuantitativos y cualitativos de las canales, ségun los sistemas de produccíon. Cuad. INIA, 1988. v.17, p.19-41.

SAINZ, R.D. Qualidade das carcaças e da carne ovina e caprina. In REUNIÃO ANUAL DA SOCIEDADE BRASILEIRA DE ZOOTECNIA, 33., SIMPÓSIO INTERNACIONAL SOBRE TÓPICOS ESPECIAIS EM ZOOTECNIA, 1996, Fortaleza. Anais... Fortaleza: Sociedade Brasileira de Zootecnia, 1996. p.3-14.

SANTOS, C.L. Estudo do desempenho, das características da carcaça e do crescimento alométrico de cordeiros das raças Santa Inês e Bergamácia. Lavras: Universidade Federal de Lavras, 1999. 142p. Dissertação (Mestrado em Zootecnia) Universidade Federal de Lavras, 1999.

STATISTICAL ANALYSIS SYSTEM - SAS. SAS user's guide: statistics. 5.ed. Cary: 1995. 1290p.

SILVA, L.F.; PIRES, C.C.; SILVA, J.H.S. et al. Crescimento de cordeiros abatidos com diferentes pesos. Osso, músculo, gordura da carcaça e de seus cortes. Ciência Rural, v.30, n.4, p.671675, 2000a.

SILVA, L.F.; PIRES, C.C.; ZEPPENFELD, C.C. et al. Crescimento de regiões da carcaça de cordeiros abatidos com diferentes pesos. Ciência Rural, v.30, n.3, p.481-484, 2000b.

SILVA, S.J.; PORTUGAL, A.V. The effect of weight on growth and carcass quality of Serra da Estrela and Merino Branco lambs raised in intensive production system. Revista Portuguesa de Zootecnia, v.7, n.1, p.109-129, 2000. 